\title{
Part orientation optimisation for the additive layer manufacture of metal components
}

\author{
H. D. Morgan ${ }^{1}$ (I) - J. A. Cherry ${ }^{1}$ - S. Jonnalagadda ${ }^{2}$ - D. Ewing ${ }^{2} \cdot$ J. Sienz ${ }^{1}$
}

Received: 20 April 2015 / Accepted: 23 November 2015 / Published online: 11 January 2016

(C) The Author(s) 2016. This article is published with open access at Springerlink.com

\begin{abstract}
In a number of additive layer manufacturing processes, particularly for metals, additional support structure is required during the build process to act as scaffolding for overhanging features and to dissipate process heat. Such structures use valuable raw materials and their removal adds to post processing time. The objective of this study was to investigate whether a simple, single objective optimisation technique could be used to find the best orientation of the part, that would minimise the volume of support needed during the build. Not only reducing waste but potentially providing an effective and consistent approach for inexperienced users to orient components during manufacture. Software was developed using MatLab with an unconstrained optimisation algorithm implemented to search the different rotations of the part and identify the configuration with the least requirement for support volume. The algorithm was gradient based, and so multiple starting points were used to identify a global minimum. The efficacy of the algorithm is illustrated with three different case studies of increasing complexity. Additionally, the component of the final study was manufactured, which allowed a comparison between the algorithm's results and the orientations chosen by experienced operatives. In two of the three case studies, the software was able to find good solutions for the support volume minimisation. For the manufactured part,
\end{abstract}

$\triangle$ H. D. Morgan

h.d.morgan@swansea.ac.uk

1 ASTUTE, College of Engineering, Swansea University, Swansea, UK

2 Renishaw plc, Stone, Staffordshire, UK only one of the results matched the orientation chosen by the operators, the other was orientated in a similar way but the difference added significantly to the required support volume. Future developments of the software would benefit from incorporating the expertise of the manufacturing operative.

Keywords Optimisation · Additive manufacture $\cdot$ Part orientation $\cdot$ SLM

\section{Introduction}

Additive layer manufacture (ALM), the process of building components in layers directly from a computer-aided design (CAD) geometry, has made increasing progress in both polymer and metal manufacture. Refinements to the process to improve production efficiency and accuracy have been studied in some detail over the past 20 years $[1,4,12$, 13]. The majority of the published research has focussed on plastic technologies. This paper describes research aimed at optimising the performance of metallic processes, e.g. selective laser melting (SLM) and direct metal laser sintering (DMLS); but, will first review some of the lessons learnt from other processes and materials.

Considering the work of Phatak and Pande [14], five distinct parameters were identified for fused deposition modeling (FDM) in a multi-objective optimisation, namely:

- Surface roughness

- Interior material

- The volume of the support structure

- Contact surface area

- Build height

The surface roughness measure used was the degree of 'staircasing' present in any particular orientation. Adjacent 


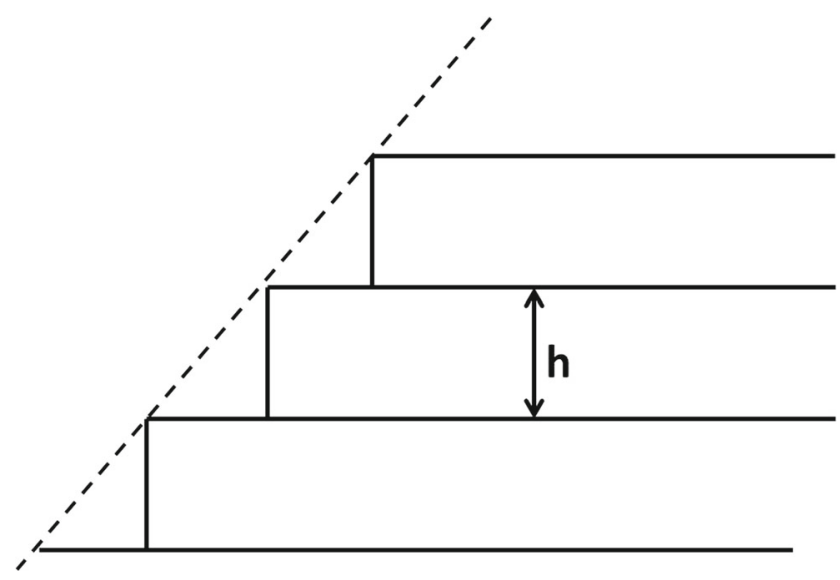

Fig. 1 Staircasing effect caused by adjacent layers of material of height ' $h$ '

layers create a non smooth stepping effect when building an inclined plane (see Fig. 1). In FDM, the layer thickness, $\mathrm{h}$, is of the order of $200 \mu \mathrm{m}$; at least four times the thickness typical of SLM. A number of authors [11, 15, 20] use adaptive slicing to reduce this effect, using thinner layers in more critical areas. However, adaptive slicing is not readily available in all ALM technologies, and although it may be effective when building a single part, it becomes increasingly complex for multiple parts built simultaneously.

In metal ALM, staircasing may not be the dominant factor in terms of roughness. Maximum powder particle diameter can be larger than the layer thickness (e.g. $60 \mu \mathrm{m}$ powder compared to $50 \mu \mathrm{m}$ slice thickness) and so entrainment at the surface of partially sintered powder may have a greater impact than staircasing [17].

The surface roughness creates multiple stress concentrations which can have a negative effect on the fatigue performance of the component [6]. Research in this area indicates that post processing is necessary to increase fatigue life, particularly for titanium alloys. This may include machining to improve surface quality, heat treatment to reduce residual stresses and hot isostatic pressing (HIP) cycles to minimise internal defects [9, 22].

Most commonly, metal ALM parts are manufactured for the aerospace and medical industries and so post-processing is often essential, for example, to machine locally critical surfaces like those requiring close contact with other components, or channels for blood flow and other sensitive fluids. Under these conditions, the control of the as-built surface roughness although still important takes a lower priority. For some medical applications, high levels of surface roughness are desirable to improve bonding between an implant and living tissue, for example [5].
The freedom to reduce interior material or simply hollow out a component is more applicable to plastic parts than metals. For metal parts, strength tends to be more critical and so material removal cannot be undertaken without considering the structural integrity of the component.

Support material is required to act as scaffolding for overhanging features of design. Any parts that are inclined at a relatively small angle to the horizontal are not selfsupporting and so additional structures must be added temporarily to hold the feature in place until solidification occurs. The support material also acts as a heat sink, increasing the conduction from the melt-pool to the substrate [7]. The required angle at which this is needed varies according to the process used and can range from as low as $20^{\circ}$ [19], with a more typical range being $30-45^{\circ}[2,21]$ to the horizontal. The inclusion of supports uses additional raw material and creates waste which is difficult to recycle [3]. This increases the time and costs of building the component and also the degree of post processing required to remove the supports. Distortion of the part caused by residual stress can also be reduced by using appropriate supports [21]. The area where the support contacts the part tends to show high roughness values, but this too can be reduced at post processing.

Changing a part's orientation to reduce the support volume may increase the build height and consequently the time taken to manufacture. Some authors [12, 13] have undertaken complex, multi-objective optimisation to find solutions that allow for the impact of surface roughness, part orientation and build height. In the data available to the authors, it is not clear that there is a significant correlation between build height and manufacturing time. Thus, from the factors considered by Phatak and Pande [14], the optimisation of support volume remains one of the most critical factors for improving the efficiency of metallic ALM processing.

A number of authors have proposed methods of design which completely avoid the need for support structures [3, 8], but these techniques are in their infancy and have only been tested on 2D structures in the literature. Strano et al. [16] optimised the support volume for 3D structures by calculating the support at every $5^{\circ}$ rotational angle about the $\mathrm{x}$ and $\mathrm{y}$ axes, subsequently choosing the lowest value. This technique was also used with polymer ALM by Masood et al. [10]. However, this systematic approach may not find the most optimum orientation with a $5^{\circ}$ resolution, particularly when considering very complex structures. Increasing the resolutions can make solving the problem very time consuming.

This paper will present a simple single objective optimisation of the build orientation of the part by minimising the 
support structure only. Using an unconstrained optimisation algorithm in MatLab, inexperienced operators can find an effective positioning of parts for ALM build. The use of the software provides greater accuracy and consistency than other methods and the lack of complexity in the approach enables computationally efficient solutions.

The work focusses solely on the requirements of metal powder bed SLM but may prove useful to other ALM processes.

\section{Methodology}

\subsection{Support volume calculation}

Figure 2 shows a flowchart of the optimisation software for minimising the support volume. The script reads the geometry in the form of a sterolithography (stl) file. The stl file represents the surfaces of the geometry in terms of nonoverlapping triangular faces. The coordinates and order of the vertices of the triangles are obtained as well as the normal vectors for each face. Testing the angle of the normal

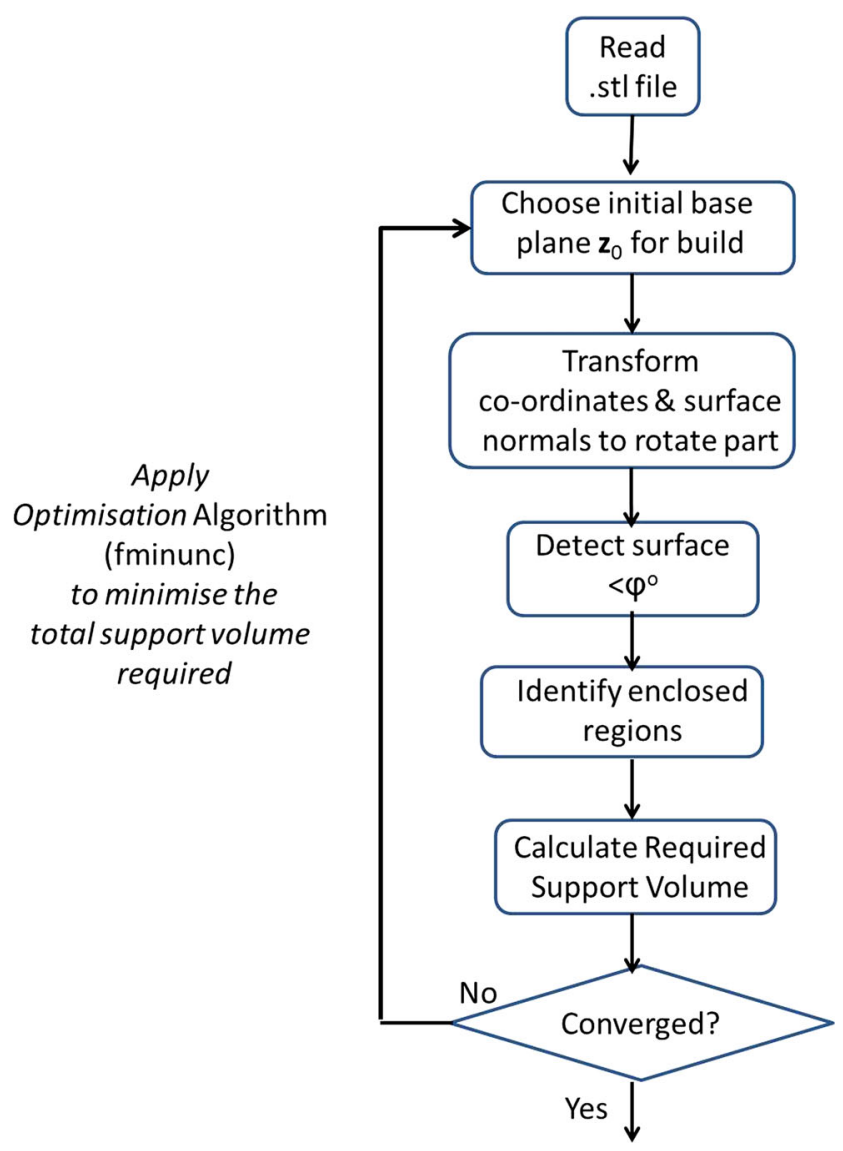

Fig. 2 Flowchart for optimisation script for part orientation

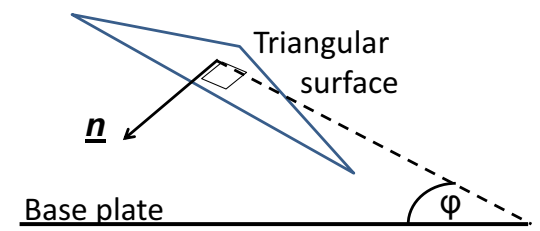

Fig. 3 Single triangular surface from the stl file showing angle to the base plate

$(\pi / 2-\phi)$ for each face relative to the building base-plate identifies those areas of the part that require support (see Fig. 3). The critical value of $\phi$ is dependent on the ALM process and equipment being used. The value taken in these calculations was $45^{\circ}$; this is the minimum angle recommended for the Renishaw AM250 SLM, which is used in each of the test cases of this paper. For each of the triangles where $\phi$ is less than $45^{\circ}$, the volume of support required was obtained from the irregular triangular prism formed as the triangle was projected perpendicular to the base plate (see Fig. 4). The three vertices $v_{i}$ give the coordinates for the calculation of the base area $B$. The vertical heights $z_{i}$ for $i=1,2,3$, can then be used to calculate the volume of the prism.

$V=\left(z_{1}+z_{2}+z_{3}\right) \frac{B}{3}$

For some triangles, other surfaces of the component lie within the field of view of the base, and so it is sufficient to have support only from those surfaces to the next feature. The total support volume takes account of this in the

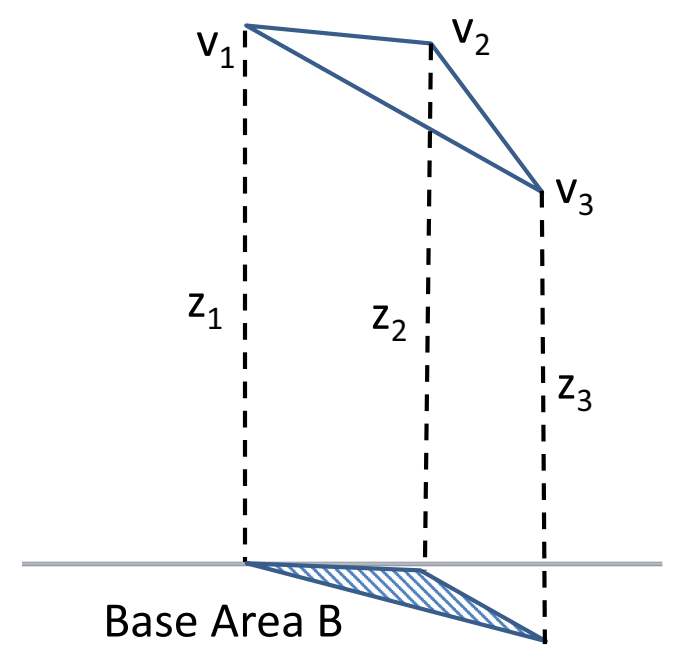

Fig. 4 Illustration of support volume calculated from projection of triangle to build plate 
calculation. It is important to note that the calculated support volume considers only geometric factors. It does not assess the need for additional support based on the internal stresses generated during the build nor the requirement for heat dissipation.

\subsection{Optimising part orientation}

In order to determine the optimum orientation for building the part while minimising the support material, the volume calculation was incorporated into an optimisation function in MatLab.

The optimisation problem was unconstrained and can be simply stated as:

min $: f(\mathbf{z})=$ Total SupportVolume

where the design variable, $\mathbf{z}$, was the normal of the base plane of the build. An initial estimate $\mathbf{z}_{0}$ of the design variable was made. This was the normal vector for the new base-plate. A new orthogonal coordinate system was generated from $\mathbf{z}_{0}$ and the co-ordinates and surface normals from the stl file were transformed to determine the new position of the part. Effectively, the part was being rotated while maintaining the $x-y$ plane as the base plate. When necessary, the rotated part was also translated to ensure that all features lay above the base plate. Following the flow of Fig. 2 , the surfaces needing support were identified and the volume of support material was calculated, making allowance for those surfaces where the support needed to extend only to the next feature.

Using the unconstrained optimisation function, fminunc, in Matlab, the value of the design variable continued to be modified until the total support volume converged to a minimum. No information was available regarding the behaviour of the gradients of this problem; consequently, fminunc defaulted to a line search algorithm. The final design variable vector was generated together with the transformation matrix, $U_{o p t}$ which transformed the original orientation to the calculated optimum one. Since the $U_{o p t}$ transformations preserved all vector lengths, then the inverse of the transformation matrix was equal to its transpose, $\left(U_{o p t}\right)^{T}$, called $V_{\text {opt }}$. This could be expressed as the product of three rotations about the $x, y$ and $z$ axes, with angles $\alpha, \beta$ and $\gamma$, respectively, i.e.

$$
\begin{aligned}
& V_{o p t}= \\
& \quad\left(\begin{array}{ccc}
\cos \gamma & -\sin \gamma & 0 \\
\sin \gamma & \cos \gamma & 0 \\
0 & 0 & 1
\end{array}\right)\left(\begin{array}{ccc}
\cos \beta & 0 & \sin \beta \\
0 & 1 & 0 \\
-\sin \beta & 0 & \cos \beta
\end{array}\right)\left(\begin{array}{ccc}
1 & 0 & 0 \\
0 & \cos \alpha & -\sin \alpha \\
0 & \sin \alpha & \cos \alpha
\end{array}\right)
\end{aligned}
$$

Multiplying the matrices and comparing terms gave solutions for $\alpha$ and $\beta$, which were sufficient for orienting the part in the ALM equipment. The rotation $\gamma$ about the $z$ axis was not needed as the support volume was independent of this rotation.

As the line search employs a gradient-based method, a global solution could not be guaranteed in this optimisation. In an attempt to compensate for this, multiple starting points were used to improve the likelihood of identifying the best optimum.

\section{Results and discussion}

\subsection{Support volume accuracy}

The Renishaw AM250 SLM equipment used Marcam Engineering AutoFAB software to set up the part orientation and manufacturing parameters. Using stl files for a number of different components, a comparison was made between the support volume calculated by the AutoFAB software and the MatLab calculations. The results are shown in Fig. 5. The graph shows a good linear correlation between the two sets of data, with the MatLab calculation being typically, approximately three times higher than the AutoFAB. The AutoFAB supports are not completely dense, typically $1 \mathrm{~mm}$ support strands are separated by $2 \mathrm{~mm}$ gaps (see Fig. 6), which may account for the gradient of the trend line being close to one third.

There is some known loss of accuracy in the script calculations:

(i) The triangular surfaces of the stl file give an approximation to any curved surfaces. The degree of error can be reduced by increasing the resolution of the

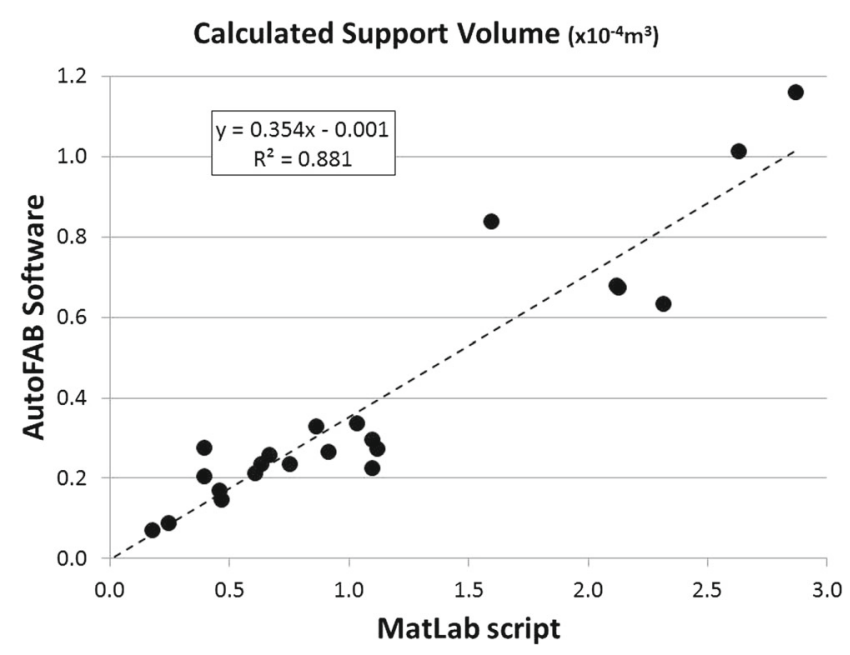

Fig. 5 Comparison of volume of support material calculated by AutoFAB and MatLab optimisation software 


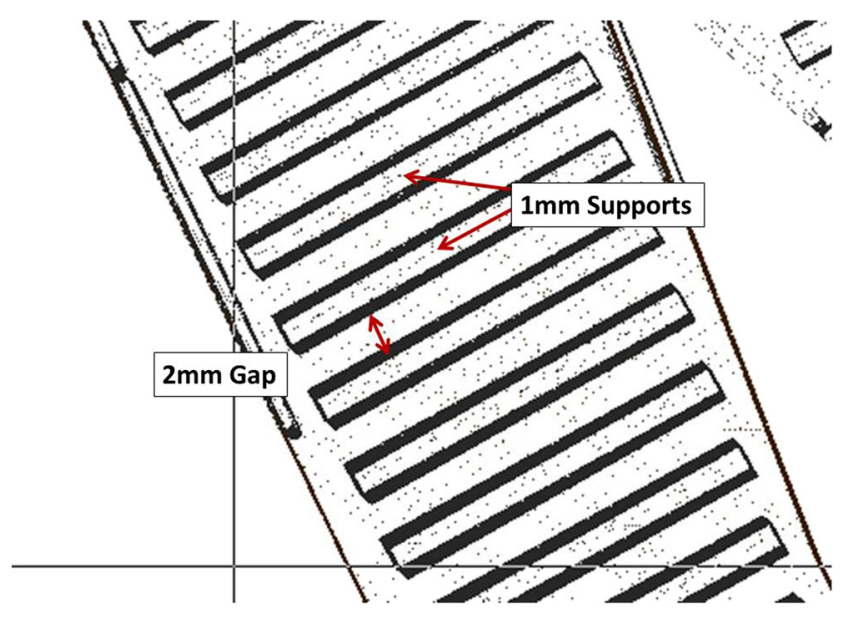

Fig. 6 Example of AutoFAB support structure in cross section showing $1 \mathrm{~mm}$ supports with a $2 \mathrm{~mm}$ gap

file when created; however, increasing the number of surfaces increases the time taken to find an optimum.

(ii) A very simplistic approach has been used to test whether the support should extend to the base of the build or only to the next feature of the part below. The script used an algorithm for the intersection of a $3 \mathrm{D}$ ray with a triangle [18] to determine if the centroid of the face lies within the triangle of any of the surfaces at a lower height. However, there are many likely configurations where the centroid may indeed lie within the lower triangle but the upper triangle may only partially overlap the one below or vice versa. When this is the case, the volume calculation would be in error. Testing all the possible options would add considerably to the computational time and so has not been considered.

Despite these observations, the support volume predictions were considered more than adequate for purpose and the optimisation script has been trialled on a number of test cases.

\subsection{Cylindrical half pipe}

Initial tests were undertaken using the simple geometry of half a cylindrical pipe (see Fig. 7). The pipe was $0.06 \mathrm{~m}$ long, with an outer diameter of approximately $0.1 \mathrm{~m}$ and wall thickness of $0.0145 \mathrm{~m}$, consisting of only six surfaces. The stl file for the pipe had 252 triangular faces. An initial calculation of the support required in the orientation shown below was $9.9 \times 10^{-5} \mathrm{~m}^{3}$.

Twenty different starting points for the optimisation were generated using Latin Hypercube sampling. Each of the three components of the initial base plate normal vector were sampled over the range -1 to +1 . The best solution found required no support volume for the part. The

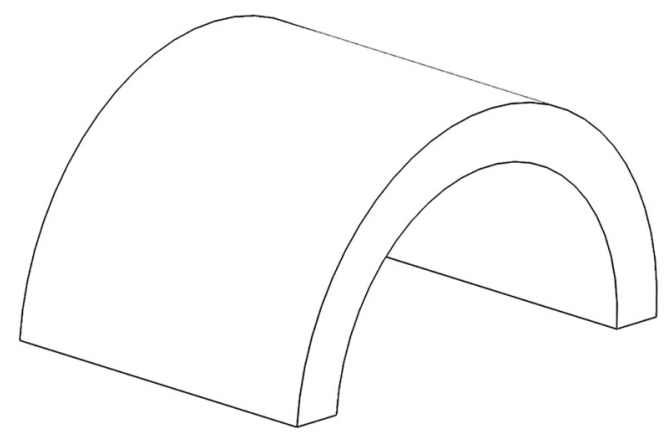

Fig. 7 Half a cylindrical pipe

optimised plane had unit normal $[0,-1,0]$,i.e. the $x-z$ plane. The orientation of the optimised part is shown in yellow in Fig. 8, together with the original part in cyan. The optimisation algorithm had successfully chosen the best orientation. This solution was found by 11 of the 20 starting points, though in 3 of these the optimised normal vector was $[0,1,0]$ confirming that when the pipe was rotated $180^{\circ}$ about the $y$-axis, there is also an optimum solution. The fastest of these solutions converged in 13 iterations.

\subsection{Engine bracket}

The second, more complex test case shown in Fig. 9 was a jet engine bracket composed of 110 surfaces which generated 6208 triangular faces in the stl file. The best solution found using multiple starting points was an optimised support volume of $0.88 \times 10^{-5} \mathrm{~m}^{3}$. The solution was found in 11 iterations with 848 function counts.

The AutoFAB software offers two default orientations, namely 'least height', where the part requires the smallest number of layers, and 'least footprint', where the area of the projection of the part on the base is at a minimum. For

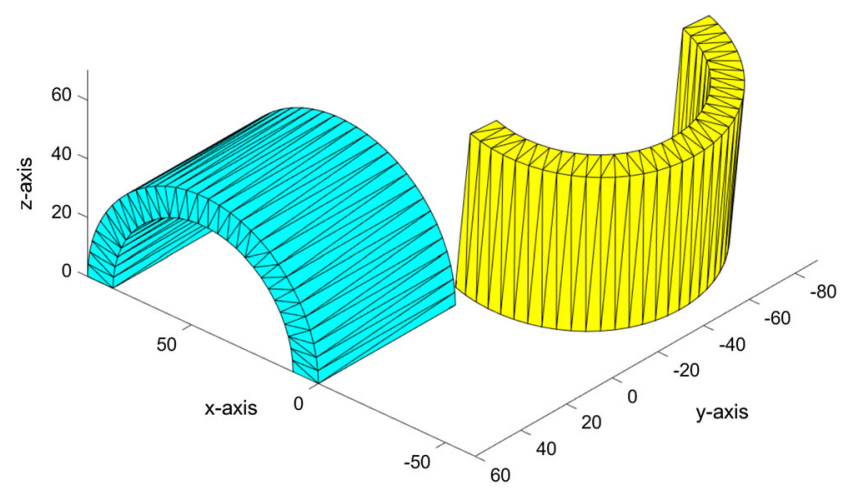

Fig. 8 Optimised solution for half pipe (yellow) compared to original orientation (cyan) 


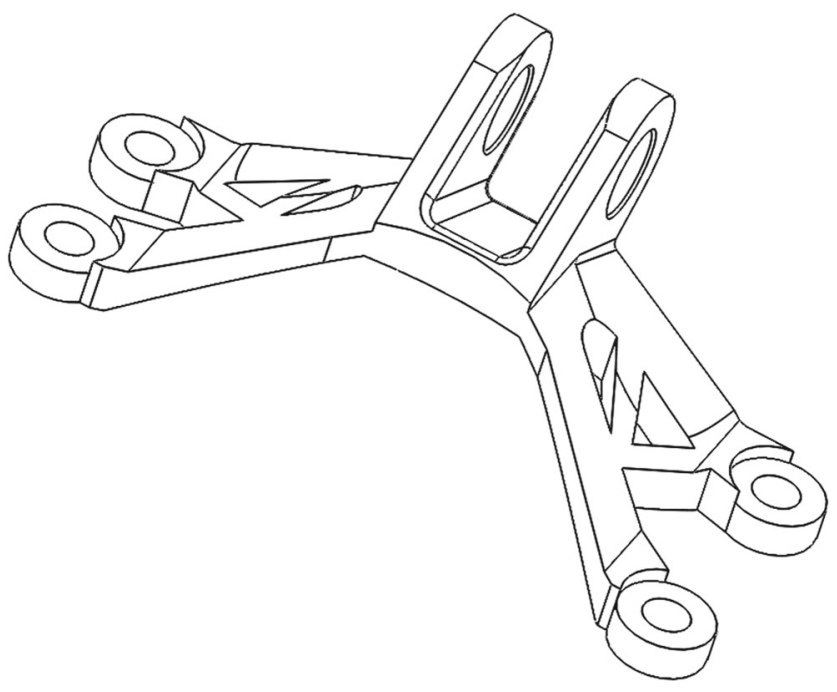

Fig. 9 Jet engine bracket design

this bracket design, the fully dense support volume for these orientations was $8.75 \times 10^{-5} \mathrm{~m}^{3}$ and $14.44 \times 10^{-5} \mathrm{~m}^{3}$ for least height and least footprint, respectively. The optimised result gave a $90 \%$ reduction in support volume over the best of these two orientations.

\subsection{Instrument casing section}

The third case study was the orientation of a casing design for an electronic component. This casing was manufactured independently by Renishaw and the as-built parts are shown in Fig. 10. Data was provided detailing the support

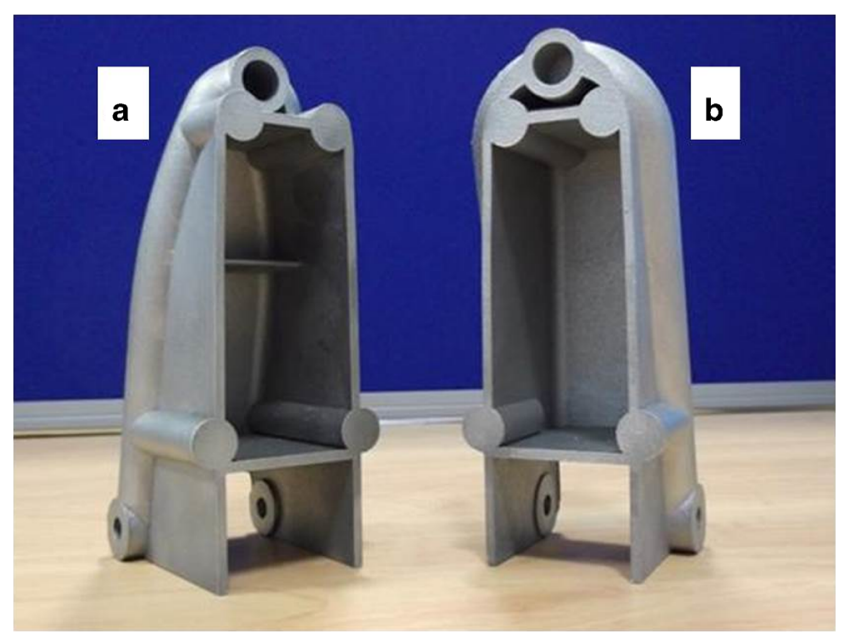

Fig. 10 ALM build casing parts showing two distinct halves a and $b$

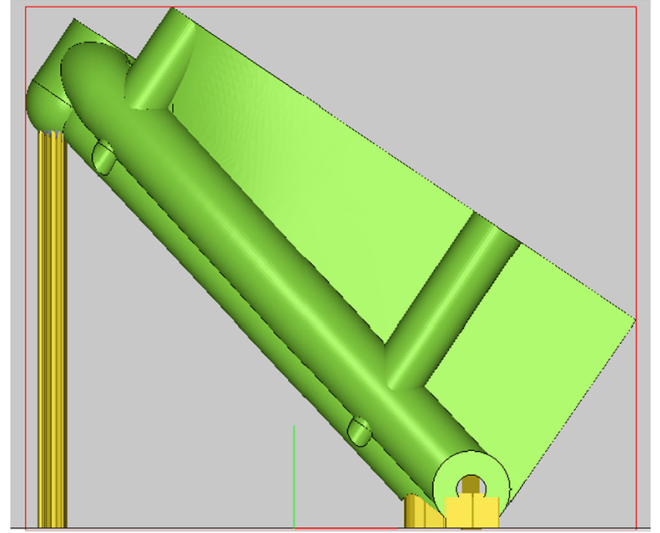

Fig. 11 Part A-best orientation from MatLab script shown in AutoFAB software with support added

structures used in the build. This has enabled the results of the optimisation software to be assessed against the build orientation chosen by experienced operators.

Part A The best orientation found by the MatLab optimisation software indicated a rotation of $-56^{\circ}$ about the $y$-axis only. This gave a total support volume of $1.57 \times 10^{-5} \mathrm{~m}^{3}$. Using these rotational angles, the volume calculated by the AutoFAB software was $0.359 \times 10^{-5} \mathrm{~m}^{3}$ with an estimated build time of $33.47 \mathrm{~h}$. The ratio of AutoFAB to MatLab support was 0.229 somewhat less than the gradient of the line of best fit in Fig. 5. Figure 11 shows the stl file for Part A with the support structure attached in AutoFAB.

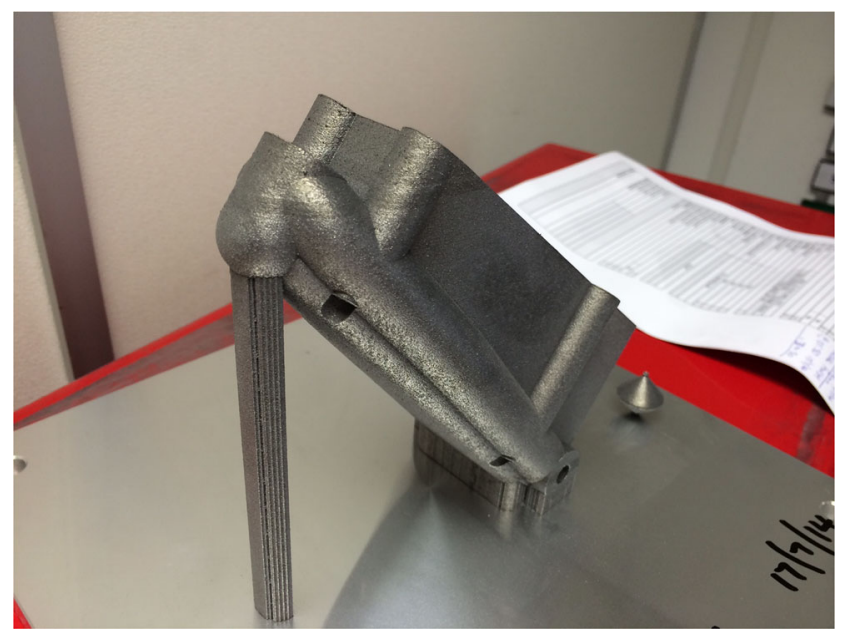

Fig. 12 ALM part a affixed to base with support material still attached 
Table 1 Comparison of support volume predictions with manufactured volumes

\begin{tabular}{|c|c|c|c|c|}
\hline & \multirow[b]{2}{*}{$\begin{array}{l}\text { Best optimum } \\
\text { using } \\
\text { MatLab } \\
\text { script }\end{array}$} & \multicolumn{3}{|c|}{ In AutoFAB } \\
\hline & & $\begin{array}{l}\text { Using } \\
\text { best } \\
\text { MatLab } \\
\text { orient. }\end{array}$ & $\begin{array}{l}\text { Using } \\
\text { Renishaw } \\
\text { orientation }\end{array}$ & $\begin{array}{l}\text { Percent } \\
\text { improve. } \\
\text { by } \\
\text { Renishaw }\end{array}$ \\
\hline \multicolumn{5}{|c|}{ Support volume $\left(\times 10^{-5} \mathrm{~m}^{3}\right)$} \\
\hline Part A & 1.57 & 0.359 & 0.33 & $8 \%$ \\
\hline Part B & 2.53 & 0.848 & 0.196 & $77 \%$ \\
\hline \multicolumn{5}{|c|}{ Time to build (h) } \\
\hline Part A & & 33.47 & 34.17 & $-2 \%$ \\
\hline Part B & & 37.7 & 36.83 & $2 \%$ \\
\hline
\end{tabular}

Stl files of the actual support material used were supplied by Renishaw after the build was completed. A photograph of part A with the support material in place is shown in Fig. 12. The positioning of the part is equivalent to a rotation of 0 and $-46^{\circ}$ about the $x$ - and $y$-axes, respectively. Subsequently, the support volume calculated for the built part by AutoFAB was $0.33 \times 10^{-5} \mathrm{~m}^{3}$, with a build time of $34.17 \mathrm{~h}$. The improvement in support volume was $8 \%$, with $2 \%$ increase in the build time, as compared to the MatLab optimisation result.

Part B A similar exercise was carried out for part B. All the results are summarised in Table 1. The predicted optimum occurred at rotational angles of $18^{\circ}$ about the $x$-axis and $45^{\circ}$ about the $y$ (see Fig. 13). When built, however, this part was rotated $45^{\circ}$ about the $y$-axis only and required only the support material shown (see Fig. 14).

In summary, it can be seen that for part A, the orientation predicted by the algorithm was similar to the build. Only a small reduction in support volume was achieved with the manufactured part and this took slightly longer to build. For part B, the optimiser did not find the best solution. The tilt of the part by just $18^{\circ}$ from the base plate about the $x$ axis made a very large difference to support volume, with little impact on the build time.
Fig. 13 Optimal build orientation for part $\mathrm{B}$, on right original orientation (cyan), optimum on left (yellow)

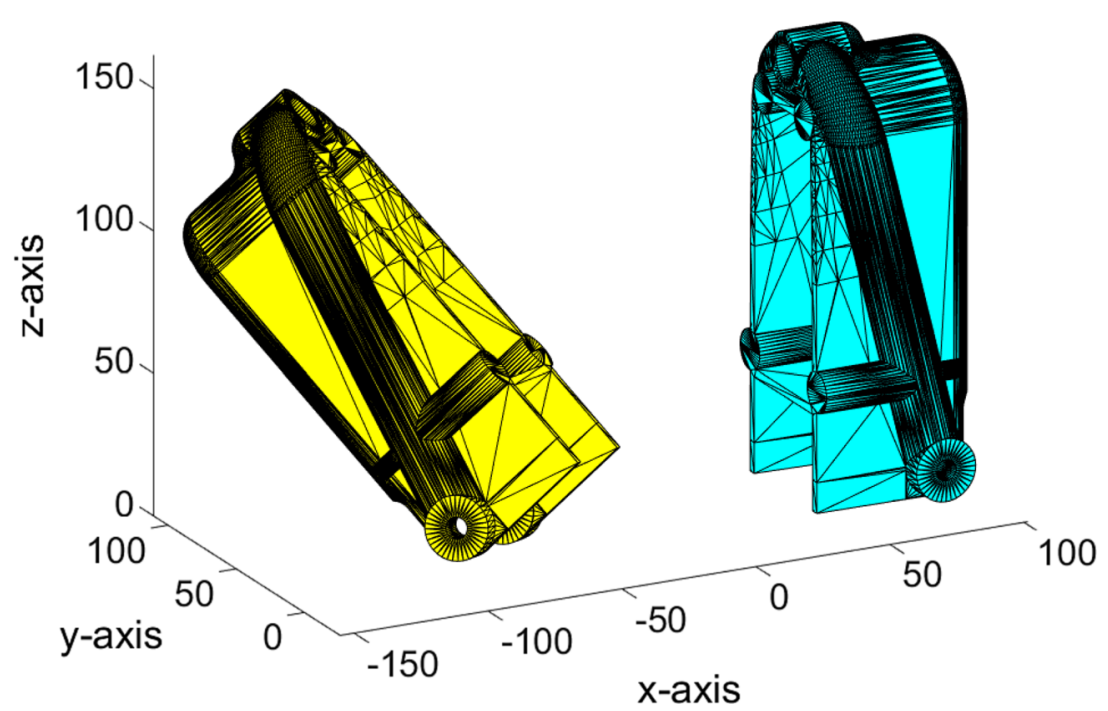




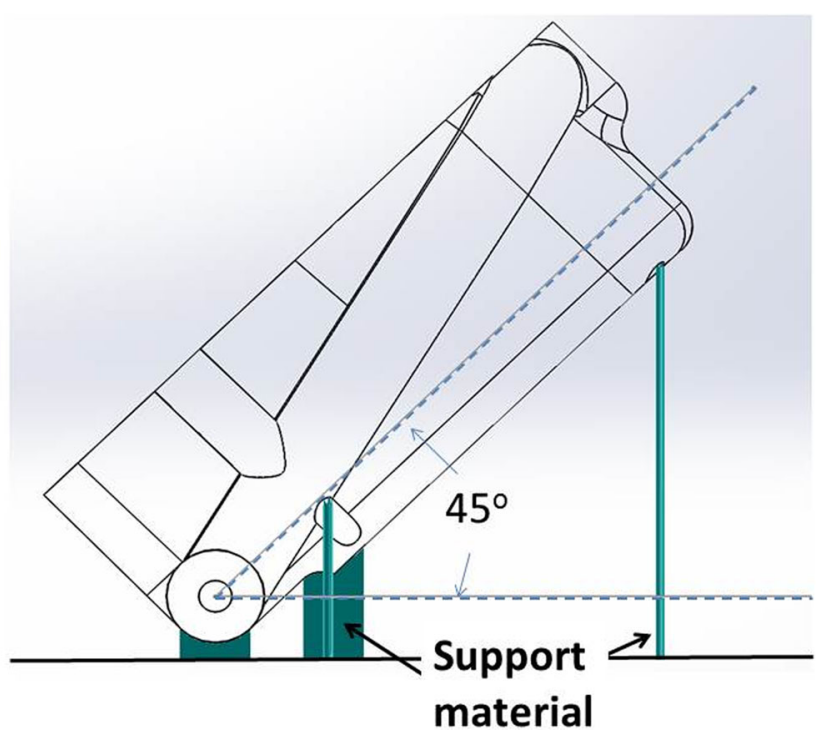

Fig. 14 Geometry of part B in as-built position showing support material attached

\subsection{Computational efficiency}

Figure 15 shows the time taken to find the best orientation of a series of parts minimising the support volume. The data is plotted as a function of the number of triangular surfaces formed in the stl file for the component. The graph shows a strong positive correlation; though in reality, the time is more closely related to the number of downward facing surfaces in any orientation, with these not easily predicted from the initial file. The graph, therefore, gives a good indication of the expected time to solution.

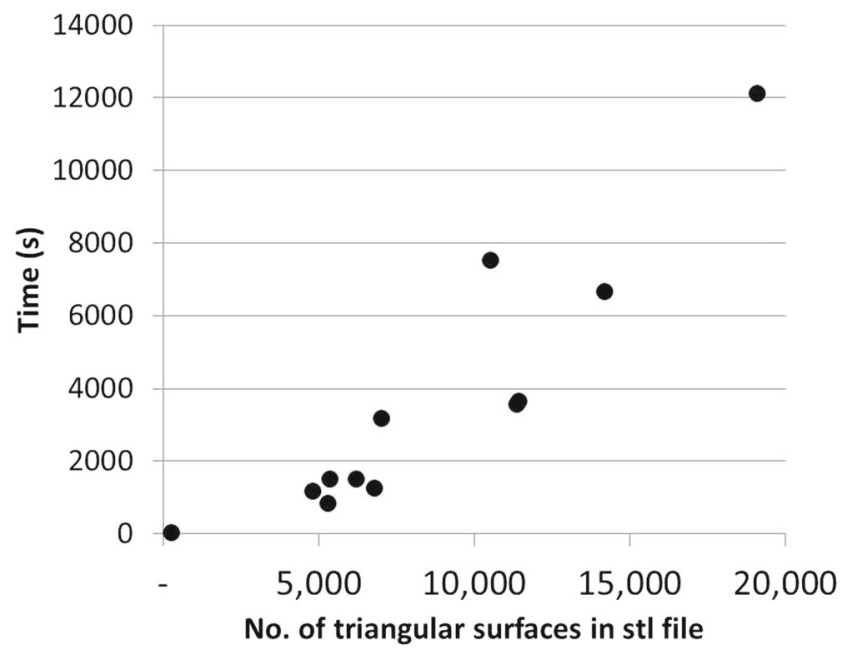

Fig. 15 Time taken to find global optimised build orientation solutions for different geometries

\section{Conclusions}

Software has been developed to predict the optimal orientation for minimising support volume in SLM or DMLS additive layer manufacture. The procedure has been tested on three different geometries and shown to bring improvements over the standard orientations recommended by preprocessing software. When compared to the build positioning selected by experienced operators, the software did not always find the best result. Although this code would enable consistent and efficient builds to be undertaken by inexperienced users, incorporating the expertise of the manufacturers in future developments would further enhance its efficacy.

Acknowledgments The authors would like to acknowledge the support of the Advanced Sustainable Manufacturing Technologies (ASTUTE) project, which is part funded from the EU's European Regional Development Fund through the Welsh European Funding Office, in enabling the research upon which this paper is based

Open Access This article is distributed under the terms of the Creative Commons Attribution 4.0 International License (http:// creativecommons.org/licenses/by/4.0/), which permits unrestricted use, distribution, and reproduction in any medium, provided you give appropriate credit to the original author(s) and the source, provide a link to the Creative Commons license, and indicate if changes were made.

\section{References}

1. Alexander P, Allen S, Dutta D (1998) Part orientation and build cost determination in layered manufacturing. Comput Aided Des 30(5):343-356

2. Atzeni E, Salmi A (2015) Study on unsupported overhangs of alsi10mg parts processed by direct metal laser sintering (dmls)

3. Brackett D, Ashcroft I, Hague R (2011) Topology optimization for additive manufacturing. In: Proceedings of the 22nd Annual International Solid Freeform Fabrication Symposium

4. Canellidis V, Giannatsis J, Dedoussis V (2009) Geneticalgorithm-based multi-objective optimization of the build orientation in stereolithography. Int J Adv Manuf Technol 45(7-8):714730

5. Chan K, Koike M, Mason R, Okabe T (2013) Fatigue life of titanium alloys fabricated by additive layer manufacturing techniques for dental implants. Metall Mater Trans A 44(2):1010-1022

6. Greitemeier D, Dalle Donne C, Syassen F, Eufinger J, Melz T (2015) Effect of surface roughness on fatigue performance of additive manufactured ti6al4v. Mater Sci Technol 0(0)

7. Kruth J, Mercelis P, Van Vaerenbergh J, Craeghs T (2007) Feedback control of selective laser melting. In: 3rd International Conference on Advanced Research in Virtual and Rapid Prototyping 
8. Leary M, Merli L, Torti F, Mazur M, Brandt M (2014) Optimal topology for additive manufacture: a method for enabling additive manufacture of support-free optimal structures. Mater Des 63(0):678-690

9. Leuders S, Thne M, Riemer A, Niendorf T, Trster T, Richard H, Maier H (2013) On the mechanical behaviour of titanium alloy tial6-v4 manufactured by selective laser melting: fatigue resistance and crack growth performance. Int J Fatigue 48:300-307

10. Masood S, Rattanawong W, Iovenitti P (2003) A generic algorithm for a best part orientation system for complex parts in rapid prototyping. J Mater Process Technol 139(13):110-116

11. Neeraj Panhalkar RP, Anandr S Increasing part accuracy in additive manufacturing processes using a $\mathrm{k}-\mathrm{d}$ tree based clustered adaptive layering

12. Padhye N, Deb K (2011) Multiobjective optimisation and multi?criteria decision making in sls using evolutionary approaches. Rapid Prototyp J 17(6):458-478

13. Pandey P, Thrimurthulu K, Reddy N (2004) Optimal part deposition orientation in FDM by using a multicriteria genetic algorithm. Int J Prod Res 42(19):4069-4089

14. Phatak AM, Pande S (2012) Optimum part orientation in rapid prototyping using genetic algorithm. J Manuf Syst 31(4):395-402. Selected Papers of 40th North American Manufacturing Research Conference
15. Singhal SK, Jain PK, Pandey PM (2008) Adaptive slicing for sls prototyping. Computer-Aided Design and Applications 5(1-4):412-423

16. Strano G, Hao L, Everson R, Evans K (2013) A new approach to the design and optimisation of support structures in additive manufacturing. Int J Adv Manuf Technol 66(9-12):12471254

17. Strano G, Hao L, Everson RM, Evans KE (2013) Surface roughness analysis, modelling and prediction in selective laser melting. J Mater Process Technol 213(4):589-597

18. Sunday D (2012) Intersections of rays and triangles (3d). http:// geomalgorithms.com/a06-_intersect-2.html

19. Vandenbroucke B, Kruth J (2007) Selective laser melting of biocompatible metals for rapid manufacturing of medical parts. Rapid Prototyp J 13(4):196-203

20. Verma A, Tyagi S, Yang K (2014) Modeling and optimization of direct metal laser sintering process. Int J Adv Manuf Technol:1-14

21. Wang D, Yang Y, Yi Z, Su X (2013) Research on the fabricating quality optimization of the overhanging surface in slm process. Int J Adv Manuf Technol 65(9-12):1471-1484

22. Wycisk E, Emmelmann C, Siddique S, Walther F (2013) High cycle fatigue (hcf) performance of ti-6al-4v alloy processed by selective laser melting. Advanced Materials Research 816817:134-139 